\title{
Super and ultracontractive bounds for doubly nonlinear evolution equations
}

\author{
Matteo Bonforte and Gabriele Grillo
}

\begin{abstract}
We use logarithmic Sobolev inequalities involving the $p$-energy functional recently derived in [15], [21] to prove $\mathrm{L}^{p}-\mathrm{L}^{q}$ smoothing and decay properties, of supercontractive and ultracontractive type, for the semigroups associated to doubly nonlinear evolution equations of the form $\dot{u}=\triangle_{p}\left(u^{m}\right)$ (with $(m(p-1) \geq 1)$ in an arbitrary euclidean domain, homogeneous Dirichlet boundary conditions being assumed. The bounds are of the form $\|u(t)\|_{q} \leq C\left\|u_{0}\right\|_{r}^{\gamma} / t^{\beta}$ for any $r \leq q \in$ $[1,+\infty]$ and $t>0$ and the exponents $\beta, \gamma$ are shown to be the only possible for a bound of such type.
\end{abstract}

\section{Introduction}

A well-known property of the heat equation is that, denoting by $u(t)$ its solution corresponding to the initial datum $u_{0} \in \mathrm{L}^{q}$, the bound

$$
\|u(t)\|_{r} \leq C_{r, q} \frac{\left\|u_{0}\right\|_{q}}{t^{\frac{d}{2}\left(\frac{1}{q}-\frac{1}{r}\right)}}
$$

for any $r \geq q \in[1,+\infty]$. This property is called supercontractivity if $r<+\infty$ and ultracontractivity if $r=+\infty$. Although this property is essentially obvious for the heat equation on $\mathbb{R}^{n}$ (since one can use the explicit Gaussian form of the kernel representing the semigroup at hand) it is not (although it still holds) for the semigroup associated to uniformly elliptic second order differential operators and in more general contexts. It is however wellknown that bounds like (1.1) are equivalent to the validity of suitable Sobolev

2000 Mathematics Subject Classification: 35K55, 35B45.

Keywords: Doubly nonlinear evolutions, contractivity properties, asymptotics, logarithmic Sobolev inequalities. 
inequalities for the Dirichlet form associated to the generator of the evolution at hand, or even to (weaker) families of logarithmic Sobolev inequalities for such Dirichlet form. It is essential, to this end, that the semigroups considered enjoy the Markov property, a property equivalent to the fact that the (self-adjoint) generator of the evolution defines a Dirichlet form: this means in particular that they can be extended to positivity preserving contractions on each $\mathrm{L}^{p}$ space including the case $p=+\infty$. For an excellent reference on such topics, which originate from the pioneering work of L. Gross [22], we refer to [14] and to the references quoted there.

In some recent papers we have tried to extend this connection to several nonlinear settings. In particular, the heat equation associated to the $p$-laplacian and to $p$-sublaplacians corresponding to a collection of Hörmander vector fields as been dealt with in [11], [13], [7]; the cases $p>d$ and $p<d$ require an entirely different treatment, as well as the cases of Dirichlet or, respectively, Neumann boundary conditions if the equation is considered on an incomplete manifold. There, an essential tool is the recently established notion of nonlinear Dirichlet form (see [12] and the former papers [9], [3]). The other essential tools are suitable entropic type estimates and a logarithmic Sobolev inequality involving the $p$-energy functional $\mathcal{E}_{p}(u)=\int|\nabla u|^{p} \mathrm{~d} x$, an inequality which has appeared for general $p$ for the first time in [4] and has been proved in a more abstract setting in [11], [13], where it is derived as a consequence of Sobolev inequalities. Later on, such inequality has been established by [15] and [21] in $\mathbb{R}^{n}$ with sharp constants.

The porous media equation

$$
\dot{u}=\triangle\left(u^{m}\right)
$$

has been studied recently in [8] and displays similar contraction properties. Recently, the paper [17] considered the doubly nonlinear equation

$$
\dot{u}=\triangle_{p} u^{1 /(p-1)}
$$

Such equation displays special scaling properties (if $u$ is a solution then $\lambda u$ is again a solution for all positive $\lambda$ ) which make the discussion very similar to the linear case, provided existence and some qualitative property for the solution are shown and the appropriate logarithmic Sobolev inequality is used. For a proof of existence for a wider class of equations and initial data see [5], [23].

Our aim here is to consider, on an open connected domain $M \subset \mathbb{R}^{n}$, the doubly nonlinear evolution equation in the general degenerate case

$$
\dot{u}=\triangle_{p} u^{m}:=\nabla \cdot\left(\left|\nabla u^{m}\right|^{p-2} \nabla u^{m}\right), \quad(p-1) m \geq 1 .
$$


Special cases are the porous media equation $(p=2)$, the heat equation driven by the $p$-Laplacian $(m=1)$ and the case dealt with in [17] $(m=1 /(p-1))$. The sharp logarithmic Sobolev inequalities established in [15], [21] will be a crucial tool. Here, as usual in the literature, we use the convention $u^{m}=|u|^{m-1} u$. Homogeneous Dirichlet boundary conditions are assumed when $M \neq \mathbb{R}^{d}$, although they may be irrelevant if the $p$-capacity of the boundary is zero.

Several authors gave existence results for weak solutions to this problem on bounded domains (see e.g. [2], [6], [10], [28]), and in more general situations including the whole Euclidean space (see e.g. [20], [23]) and general domains (see e.g. [5]). We recall that a function $u(t, x)$ defined in $M \times(0, T)$ is a weak solution of

$$
\left\{\begin{array}{l}
\dot{u}=\triangle_{p}\left(u^{m}\right) \\
u(0, \cdot)=u_{0} \in L^{q}(M) \\
u(t, x)=0, \quad \text { if } t>0 \text { and } x \in \partial M
\end{array}\right.
$$

if

$$
\begin{gathered}
u^{m}:=|u|^{m-1} u \in \mathrm{L}^{p}\left(0, T ; W_{0}^{1, p}(M)\right) \\
-\int_{0}^{T} \int_{M}\left[-u(t, x) \dot{\varphi}(t, x)+\left|\nabla\left(u(t, x)^{m}\right)\right|^{p-2} \nabla\left(u(t, x)^{m}\right) \nabla \varphi(t, x)\right] \mathrm{d} x \mathrm{~d} t \\
=\int_{M} u_{0}(x) \varphi(0, x) \mathrm{d} x
\end{gathered}
$$

for all

$$
\varphi \in \mathrm{L}^{p}\left(0, T ; W_{0}^{1, p}(M)\right) \cap W^{1,1}\left(0, T ; \mathrm{L}^{1}(M)\right)
$$

such that $\varphi(T)=0$.

Our first result is the following:

Theorem 1.1 Let $u(t)$ be the weak solution to the problem (1.2) where $M \subseteq \mathbb{R}^{d}$ is an open connected domain and $p>1, m>0, m(p-1) \geq 1$, $q \geq 1$. Then the following supercontractive bound holds true:

$$
\|u(t)\|_{\varrho} \leq C \frac{\left\|u_{0}\right\|_{q}^{\gamma}}{t^{\alpha}}
$$

Where

$$
\begin{aligned}
& \varrho>q \geq 1 \\
& \alpha=\frac{d}{\varrho} \frac{\varrho-q}{p q+d(m(p-1)-1)} \\
& \gamma=\frac{q}{\varrho} \frac{p \varrho+d(m(p-1)-1)}{p q+d(m(p-1)-1)}
\end{aligned}
$$


Moreover the following ultracontractive bound holds true:

$$
\|u(t)\|_{\infty} \leq C \frac{\left\|u_{0}\right\|_{q}^{\gamma}}{t^{\alpha}}
$$

Where

$$
\begin{aligned}
\alpha & =\frac{d}{p q+d(m(p-1)-1)} \\
\gamma & =\frac{p q}{p q+d(m(p-1)-1)} .
\end{aligned}
$$

The exponents $\alpha, \gamma$ are the only possible for a bound like (1.3) or (1.5) to hold.

Finally the contraction property

$$
\|u(t)\|_{q} \leq\left\|u_{0}\right\|_{q}
$$

is valid for all $t>0$ and $q \in[1,+\infty]$.

Remark 1.2 (sharpness of the bounds) Besides noticing that the exponents in the above bound are the only possible, we also comment that the $\mathrm{L}^{1}-\mathrm{L}^{\infty}$ bound involves a power of $t$ which is exactly the one appearing in the well-known Barenblatt solutions in $\mathbb{R}^{n}$, both for large and small times (see e.g. [19]). It will be a consequence of the subsequent Theorem that the long time behaviour in the case of domains of finite measure is sharp as well, by comparison e.g. with the results of [27].

Remark 1.3 (the case $m=1 /(p-1)$ ) We comment further on the differences between our discussion and the one given for the special case studied in [17]. They lie in the fact that, when using the usual strategy of deriving the $\mathrm{L}^{r(s)}$ norm of the solution w.r.t. time, two separate entropic (or Young) terms of the form $\int u^{\alpha} \log u \mathrm{~d} x$ with different $\alpha$ are present in our case: one coming from the time dependence of $r$ and the other one from the time dependence of $u$ itself. The two terms have different sign and therefore they can be usefully estimated in terms of the $p$-energy functional only after a delicate analysis of the Young functionals involved. In the case $m=1 /(p-1)$ (and only in such case) the two terms correspond to the same value of $\alpha$ and hence the discussion bears no essential difference with the linear case, provided some steps which are in principle only formal are justified, a step performed in [11] by arguments adaptable to the cases at hand as well.

Remark 1.4 (The case $\boldsymbol{m}=\mathbf{1}, \boldsymbol{p}=\boldsymbol{d}$ ) The discussion of [11], [7] allowed to consider the equation $\dot{u}=\triangle_{p} u$ for $p>1$ but different from the Euclidean dimension $d$. In fact, bounds of the form (1.5) were proved there and the 
exponents $\alpha, \gamma$ converge, both as $p \uparrow d$ and as $p \downarrow d$ to the corresponding values given here: but the proportionality constant $C$ blows up in both cases, so that the methods used there did not allow to treat such case.

To investigate further the similarities (or the possible differences) between the contractivity properties of the linear heat semigroup and of the nonlinear semigroups studied here, one should recall that, if the Lebesgue measure of the domain $D$ is finite, the spectrum $\sigma(-\triangle)$ of $-\triangle$ is purely discrete and $\inf \sigma(-\triangle)=E^{0}>0$, so that the spectral Theorem implies exponential time decay of any solution as $t \rightarrow+\infty$.

A natural question is if a similar behavior is enjoyed by the solutions to the equation at hand. The answer is negative in the case $m(p-1)>1$, since it is known (see [27]) that the large time behavior is of the form

$$
t^{-1 /[(p-1) m-1]}
$$

at least if the domain and the datum are sufficiently smooth. In the same paper it is shown also that in the case $m(p-1)=1$ the solution decays exponentially, at least if both the data and the bounded domain are sufficiently smooth. The bounds are sharp in both situations. Clearly the latter bounds are sharper than what we stated in Theorem 1.1 if $t$ is large and it is in this sense only that the finite measure assumption improves the time decay.

We now state our second result, whose proof relies essentially on the validity of Theorem 1.1. It holds for general domains with finite measure and all initial data without any smoothness assumption.

Theorem 1.5 Let $u(t)$ be the weak solution to the problem (1.2) where $M \subseteq \mathbb{R}^{d}$ is an open connected domain with finite measure and $p>1, m>0$, $m(p-1) \geq 1, q \geq 1$. Then the following holds:

- if $m(p-1)>1$ then the following ultracontractive bound holds true for any $t \geq 1$ :

$$
\|u(t)\|_{\infty} \leq \frac{K_{1}}{t^{\alpha}} \frac{1}{\left(C^{\prime} t+\left\|u_{0}\right\|_{q}^{-(m(p-1)-1)}\right)^{\gamma /(m(p-1)-1)}}
$$

where

$$
\begin{aligned}
\alpha & =\frac{d}{p q+d(m(p-1)-1)} \\
\gamma & =\frac{p q}{p q+d(m(p-1)-1)}
\end{aligned}
$$


116 M. Bonforte and G. Grillo

In particular for any $t \geq 1$ we have the absolute bound:

$$
\|u(t)\|_{\infty} \leq \frac{K_{2}}{t^{1 /(m(p-1)-1)}} ;
$$

- if $m(p-1)=1$ then the following ultracontractive bound

$$
\|u(t)\|_{\infty} \leq K_{3} \mathrm{e}^{-\sigma t}\|u(0)\|_{q}
$$

holds for any $t \geq 1$, where

$$
\sigma=\frac{(r-1) m^{p-1} p^{p}}{r^{p+1}} \lambda_{p}
$$

and $\lambda_{p}$ is the constant appearing in the Poincarè inequality

$$
\|f\|_{p}^{p} \leq \lambda_{p}^{-1}\|\nabla f\|_{p}^{p}, \quad f \in W_{0}^{1, p}(M) .
$$

Remark 1.6 The constant $\lambda_{p}$ has an interpretation as ground state energy of the $p$-Laplacian, see [24] and references quoted.

\section{Preliminary Results}

We start defining the following Young functional, of crucial importance in the sequel:

$$
J(r, u)=\int_{M} \log \left(\frac{|u|}{\|u\|_{r}}\right) \frac{|u|^{r}}{\|u\|_{r}^{r}} \mathrm{~d} x
$$

for any $r \geq 1$ and

$$
u \in X=\bigcap_{p=1}^{+\infty} \mathrm{L}^{p}(M) .
$$

Lemma 2.1 Let $u(t)$ be a weak solution to (1.2) corresponding to an $C_{c}^{\infty}(M)$ initial datum $u_{0}$. Then the following inequality holds true:

$$
\begin{aligned}
& \frac{\mathrm{d}}{\mathrm{d} s} \log \|u(s)\|_{r(s)}=\frac{\dot{r}(s)}{r(s)} J(r(s), u(s)) \\
& \quad-\frac{r(s)(r(s)-1) m^{p-1} p^{p}}{(r(s)+m(p-1)-1)^{p}} \frac{1}{\|u(s)\|_{r(s)}^{r(s)}}\left\|\nabla\left(|u(s)|^{\frac{r(s)+m(p-1)-1}{p}}\right)\right\|_{p}^{p}
\end{aligned}
$$


Proof. We remind the reader that the solution corresponding to an initial datum $u_{0} \in C_{c}^{\infty}(M)$ exists and is essentially bounded, when $M \subseteq \mathbb{R}^{d}$ is any open domain (not necessarily bounded). See [5] for the existence, where such result is given for even more general equations. Existence is proved in [23] as well for the case of the whole $\mathbb{R}^{d}$, for a class of initial data which is essentially optimal and larger then the class considered here. As for the $\mathrm{L}^{\infty}$ bound it follows from minor modifications of Lemma 3.2 of [23] (for the case $m(p-1)>1$ ) and Lemma 5.1 of [23] (for the case $m(p-1)=1$ ).

Now we compute:

$$
\begin{aligned}
& \frac{\mathrm{d}}{\mathrm{d} t}\|u(t)\|_{r}^{r}= \\
& \quad=r \int_{M} u(t, x)^{r-1} \dot{u} \mathrm{~d} x \\
& \quad=-r \int_{M} \nabla\left(u(t, x)^{r-1}\right) \cdot \nabla\left(u(t, x)^{m}\right)\left|\nabla\left(u(t, x)^{m}\right)\right|^{p-2} \mathrm{~d} x \\
& =-\frac{r(r-1) m^{p-1} p^{p}}{(r+m(p-1)-1)^{p}} \int_{M}\left|\nabla\left(|u(t, x)|^{\frac{r+m(p-1)-1}{p}}\right)\right|^{p} \mathrm{~d} x
\end{aligned}
$$

This property can be proved as in Lemma 3.1 of [11] according to the definition of weak solution given above, given the fact that the solution corresponding to the data presently considered is in $\mathrm{L}^{\infty}$ at all times. Therefore, by the definition of the Young functional:

$$
\begin{aligned}
& \frac{\mathrm{d}}{\mathrm{d} s} \log \| u(s) \|_{r(s)}= \\
&= \frac{\dot{r}(s)}{r(s)} \int_{M} \log \left(\frac{|u(s, x)|}{\|u(s)\|_{r(s)}}\right) \frac{|u(s, x)|^{r(s)}}{\|u(s)\|_{r(s)}^{r(s)}} \mathrm{d} x \\
& \quad+\frac{r(s)}{\|u(s)\|_{r(s)}^{r(s)}} \int_{M} u(s, x)^{r(s)-1} \dot{u}(s, x) \mathrm{d} x \\
&=\frac{\dot{r}(s)}{r(s)} J(r(s), u(s))-\frac{r(s)(r(s)-1) m^{p-1} p^{p}}{(r(s)+m(p-1)-1)^{p}} \times \\
& \quad \times \frac{1}{\|u(s)\|_{r(s)}^{r(s)}} \int_{M}\left|\nabla\left(|u(t, x)|^{\frac{r(s)+m(p-1)-1}{p}}\right)\right|^{p} .
\end{aligned}
$$

Now we need to estimate the $p$-energy term and this will be possible using a suitable logarithmic Sobolev inequality (LSI in the sequel). We will use the LSI that appears in the work of Del Pino and Dolbeault (for the case $p<d$, see [15]) or in the work of Gentil (for all $p \geq d$ and other generalizations, see [21]). These two results can be summarized in the following proposition: 
Proposition 2.2 (Del Pino-Dolbealt [15], Gentil [21]) Let $p \in(1,+\infty)$. Then for any $w \in W^{1, p}\left(\mathbb{R}^{d}\right)$ with

$$
\|w\|_{p}^{p}=\int_{\mathbb{R}^{d}}|w|^{p} \mathrm{~d} x=1
$$

we have:

$$
\int_{\mathbb{R}^{d}}|w|^{p} \log \left(|w|^{p}\right) \leq \frac{d}{p} \log \left[\mathcal{L}_{p} \int_{\mathbb{R}^{d}}|\nabla w|^{p} \mathrm{~d} x\right]
$$

with

$$
\mathcal{L}_{p}=\frac{p}{d}\left(\frac{p-1}{e}\right)^{p-1} \pi^{\frac{p}{2}}\left[\frac{\Gamma\left(\frac{d}{2}+1\right)}{\Gamma\left(d \frac{p-1}{p}+1\right)}\right]^{\frac{p}{d}}
$$

Remark 2.3 Inequality (2.5) is optimal and functions for which equality holds are known from the above mentioned papers if $p \neq 1$. For such limiting case $(p=1)$ see [4]. It will be however clear from the sequel that what will really be useful for determining the correct exponents in our bounds is the $d / p$ factor appearing in the r.h.s. of $(2.5)$, the value of the constant $\mathcal{L}_{p}$ having influence only on the value of the proportionality constant $C$ in our main Theorems.

We will use the above LSI in a slightly different form which is more useful to our purposes:

Proposition 2.4 (Logarithmic Sobolev Inequality) Let $M \subseteq \mathbb{R}^{d}$ and let $f \in W_{0}^{1, p}(M)$, with $p \in[1,+\infty)$. Then the following LSI holds true for any $\varepsilon>0$ :

$$
\|\nabla f\|_{p}^{p} \geq \frac{\|f\|_{p}^{p}}{\varepsilon \mathcal{L}_{p}}\left[\frac{p}{d} J\left(1, f^{p}\right)+\log \varepsilon\right]
$$

Proof. Just let

$$
w(x)=\frac{f(x)}{\|f\|_{p}}
$$

in the LSI (2.5) and use the definition of the Young functional (2.1) and the numerical inequality

$$
\log x \leq-\log \varepsilon+\varepsilon x
$$

In the next lemma we will use the LSI (2.6) to estimate the $p$-energy functional: 
Lemma 2.5 Let $u(t)$ be a weak solution to (1.2) corresponding to a $C_{c}^{\infty}(M)$ initial datum $u_{0}$. Then the following inequality holds true:

$$
\begin{aligned}
\frac{\mathrm{d}}{\mathrm{d} s} \log & \|u(s)\|_{r(s)} \leq \\
\leq- & \frac{\dot{r}(s)}{r(s)} \frac{d(m(p-1)-1)}{\operatorname{pr}(s)+d(m(p-1)-1)} \log \|u(s)\|_{r(s)} \\
- & \frac{\dot{r}(s)}{r(s)} \frac{d}{\operatorname{pr}(s)+d(m(p-1)-1)} \times \\
& \times \log \left(\frac{m^{p-1} p^{p}}{d \mathcal{L}_{p}} \frac{r(s)^{2}}{\dot{r}(s)} \frac{(r(s)-1)(p r(s)+d(m(p-1)-1))}{|r(s)+m(p-1)-1|^{p}}\right)
\end{aligned}
$$

Proof. The first step in the proof is to use the LSI (2.6) in the inequality (2.2) applied to the function $f=|u(s)|^{\frac{r(s)+m(p-1)-1}{p}}$ :

$$
\begin{aligned}
& \text { 2.8) } \frac{\mathrm{d}}{\mathrm{d} s} \log \|u(s)\|_{r(s)} \leq \frac{\dot{r}(s)}{r(s)} J(r(s), u(s)) \\
& -\frac{r(s)(r(s)-1) m^{p-1} p^{p}}{(r(s)+m(p-1)-1)^{p}} \frac{1}{\|u(s)\|_{r(s)}^{r(s)}}\left\|\nabla\left(|u(s)|^{\frac{r(s)+m(p-1)-1}{p}}\right)\right\|_{p}^{p} \\
& \leq \frac{\dot{r}(s)}{r(s)} J(r(s), u(s))-\frac{r(s)(r(s)-1) m^{p-1} p^{p}}{(r(s)+m(p-1)-1)^{p}} \\
& \quad \times \frac{1}{\|u(s)\|_{r(s)}^{r(s)}} \frac{\left\||u(s)|^{\frac{r(s)+m(p-1)-1}{p}}\right\|_{p}^{p}\left[\frac{p}{\varepsilon} J\left(1,|u(s)|^{\frac{r(s)+m(p-1)-1}{p} p}\right)+\log \varepsilon\right]}{\mathcal{L}_{p}} \frac{\dot{r}(s)}{r(s)} J(r(s), u(s))-\frac{1}{\varepsilon \mathcal{L}_{p}} \frac{r(s)(r(s)-1) m^{p-1} p^{p}}{(r(s)+m(p-1)-1)^{p}} \frac{\|u(s)\|_{r(s)+m(p-1)-1}^{r(s)+m(p-1)-1}}{\|u(s)\|_{r(s)}^{r(s)}} \\
& \quad \times\left[\frac{p}{d} J\left(1,|u(s)|^{r(s)+m(p-1)-1}\right)+\log \varepsilon\right]
\end{aligned}
$$

since

$$
\left\||u(s)|^{\frac{r(s)+m(p-1)-1}{p}}\right\|_{p}^{p}=\|u(s)\|_{r(s)+m(p-1)-1}^{r(s)+m(p-1)-1} .
$$

Now choosing

$$
\begin{aligned}
\varepsilon & =\frac{1}{\mathcal{L}_{p}} \frac{r(s)^{2}}{\dot{r}(s)} \frac{(r(s)-1) m^{p-1} p^{p}}{|r(s)+m(p-1)-1|^{p}} \frac{p r(s)+d(m(p-1)-1)}{d} \frac{\|u(s)\|_{r(s)+m(p-1)-1}^{r(s)+m(p-1)-1}}{\|u(s)\|_{r(s)}^{r(s)}} \\
& =\varepsilon_{1} \frac{\|u(s)\|_{r(s)+m(p-1)-1}^{r(s)+m(p-1)-1}}{\|u(s)\|_{r(s)}^{r(s)}}
\end{aligned}
$$


we obtain:

$$
\frac{\mathrm{d}}{\mathrm{d} s} \log \|u(s)\|_{r(s)} \leq \frac{\dot{r}(s)}{r(s)} J(r(s), u(s))
$$

$-\frac{\dot{r}(s)}{r(s)} \frac{d}{p r(s)+d(m(p-1)-1)}\left[\frac{p}{d} J\left(1,|u(s)|^{r(s)+m(p-1)-1}\right)\right.$

$\left.+\log \frac{\|u(s)\|_{r(s)+m(p-1)-1}^{r(s)+m(p-1)-1}}{\|u(s)\|_{r(s)}^{r(s)}}\right]-\frac{\dot{r}(s)}{r(s)} \frac{d}{\operatorname{pr}(s)+d(m(p-1)-1)} \log \varepsilon_{1}$

$\leq \frac{\dot{r}(s)}{r(s)}\left[J(r(s), u(s))+\frac{p}{d} \frac{d}{p r(s)+d(m(p-1)-1)} J\left(1,|u(s)|^{r(s)+m(p-1)-1}\right)\right.$

$$
-\frac{d(m(p-1)-1)}{\operatorname{pr}(s)+d(m(p-1)-1)} J(r(s), u(s))
$$

$$
\left.-\frac{d(m(p-1)-1)}{p r(s)+d(m(p-1)-1)} \log \|u(s)\|_{r(s)}\right]-\frac{\dot{r}(s)}{r(s)} \frac{d \log \varepsilon_{1}}{\operatorname{pr}(s)+d(m(p-1)-1)}
$$

In the last inequality we used the estimate:

$$
\begin{aligned}
& \log \frac{\|u(s)\|_{r(s)+m(p-1)-1}^{r(s)+m(p-1)-1}}{\|u(s)\|_{r(s)}^{r(s)}} \geq \\
& \quad \geq(m(p-1)-1)\left[J(r(s), u(s))+\log \|u(s)\|_{r(s)}\right]
\end{aligned}
$$

which follows from two basic facts. First, the function

$$
N(r, u)=\log \|u\|_{r}^{r}
$$

is convex with respect to the variable $r \geq 1$, so its derivative is an increasing function of $r \geq 1$. Moreover

$$
N^{\prime}(r, u)=J(r, u)+\log \|u\|_{r},
$$

so the following inequality:

$$
\begin{aligned}
N(r+m(p-1)-1, u)-N(r, u) & \geq N^{\prime}(r, u)[m(p-1)-1] \\
& =\left[J(r, u)+\log \|u\|_{r}\right](m(p-1)-1)
\end{aligned}
$$

holds if $m(p-1) \geq 1$ and leads to (2.10). 
Finally one gets:

$$
\frac{\mathrm{d}}{\mathrm{d} s} \log \|u(s)\|_{r(s)} \leq
$$

$$
\begin{aligned}
\leq & \frac{\dot{r}(s)}{r(s)}\left[\left(1-\frac{d(m(p-1)-1)}{p r(s)+d(m(p-1)-1)}\right) \frac{\dot{r}(s)}{r(s)} J(r(s), u(s))\right. \\
& \left.-\frac{p}{d} \frac{d}{p r(s)+d(m(p-1)-1)} J\left(1,|u(s)|^{r(s)+m(p-1)-1}\right)\right] \\
& -\frac{\dot{r}(s)}{r(s)} \frac{d(m(p-1)-1)}{\operatorname{pr}(s)+d(m(p-1)-1)} \log \|u(s)\|_{r(s)}-\frac{\dot{r}(s)}{r(s)} \frac{d \log \varepsilon_{1}}{\operatorname{pr}(s)+d(m(p-1)-1)} \\
= & \frac{\dot{r}(s)}{r(s)} \frac{p}{d} \frac{d}{\operatorname{pr}(s)+d(m(p-1)-1)}\left[J\left(1,|u(s)|^{r(s)}\right)-J\left(1,|u(s)|^{r(s)+m(p-1)-1}\right)\right] \\
& -\frac{\dot{r}(s)}{r(s)} \frac{d(m(p-1)-1)}{\operatorname{pr}(s)+d(m(p-1)-1)} \log \|u(s)\|_{r(s)}-\frac{\dot{r}(s)}{r(s)} \frac{d \log \varepsilon_{1}}{\operatorname{pr}(s)+d(m(p-1)-1)} \\
\leq & -\frac{\dot{r}(s)}{r(s)} \frac{d(m(p-1)-1)}{\operatorname{pr}(s)+d(m(p-1)-1)} \log \|u(s)\|_{r(s)} \\
& -\frac{\dot{r}(s)}{r(s)} \frac{d \log \varepsilon_{1}}{\operatorname{pr}(s)+d(m(p-1)-1)}
\end{aligned}
$$

where we used two further properties of the Young functional, namely:

$$
r J(r, u)=J\left(1, u^{r}\right)
$$

and

$$
J\left(1, u^{r}\right)-J\left(1, u^{r+m(p-1)-1}\right) \leq 0, \quad \text { if } m(p-1) \geq 1
$$

the proof of the first property is straightforward from the definition of the functional $J$. As for the second property, the fact that $J\left(1, u^{r}\right)$ is a nondecreasing function of $r \geq 1$ is a consequence of the convexity (w.r.t. the variable $r$ ) of the function:

$$
\phi(r, u)=\log \|u\|_{1 / r} .
$$

We refer to [1] for a proof of such fact, but comment that it is equivalent to the well known interpolation inequality:

$$
\|u\|_{1 / r} \leq\|u\|_{1 / p}^{\theta}\|u\|_{1 / q}^{1-\theta}
$$

valid when $\frac{1}{r}=\frac{\theta}{p}+\frac{1-\theta}{q}$. Now deriving $\phi$ respect to $r$ gives us:

$$
\frac{\mathrm{d}}{\mathrm{d} r} \phi(r, u)=-\frac{1}{r} J\left(\frac{1}{r}, u\right)
$$

thus, as derivative of a convex functions, $-\frac{1}{r} J\left(\frac{1}{r}, u\right)$ is non-decreasing. Now the statement follows by the definition of $\varepsilon_{1}$. 
The previous lemma gives a differential inequality for the function $y(s)=$ $\log \|u(s)\|_{r(s)}$ that we state more explicitly in the next lemma.

Lemma 2.6 Let $u(t)$ be a weak solution to (1.2) corresponding to a $C_{c}^{\infty}(M)$ initial datum $u_{0}$. Then the following differential inequality holds true for any $s \geq 0$ :

$$
\frac{\mathrm{d}}{\mathrm{d} s} y(s)+p(s) y(s)+q(s) \leq 0
$$

With

$$
\begin{aligned}
y(s)= & \log \|u(s)\|_{r(s)} \\
p(s)= & \frac{\dot{r}(s)}{r(s)} \frac{d(m(p-1)-1)}{\operatorname{pr}(s)+d(m(p-1)-1)} \\
q(s)= & \frac{\dot{r}(s)}{r(s)} \frac{d}{\operatorname{pr}(s)+d(m(p-1)-1)} \times \\
& \times \log \left(\frac{m^{p-1} p^{p}}{d \mathcal{L}_{p}} \frac{r(s)^{2}}{\dot{r}(s)} \frac{(r(s)-1)(\operatorname{pr}(s)+d(m(p-1)-1))}{|r(s)+m(p-1)-1|^{p}}\right)
\end{aligned}
$$

In particular, choosing $r(s)=\frac{\varrho-r(0)}{t} s+r(0)$, with $\varrho \geq r(0) \geq 1$, one gets the bound:

$$
y(t)=\lim _{s \rightarrow t^{-}} y(s) \leq \lim _{s \rightarrow t^{-}} y_{L}(s)=y_{L}(t)
$$

with

$$
\begin{aligned}
y_{L}(t)= & \frac{r(0)}{\varrho} \frac{p \varrho+d(m(p-1)-1)}{\operatorname{pr}(0)+d(m(p-1)-1)} y_{L}(0) \\
& -\frac{d}{\varrho} \frac{(\varrho-r(0))}{\operatorname{pr}(0)+d(m(p-1)-1)} \log (t)+C\left(p, m, d, \mathcal{L}_{p}, r(0), \varrho\right)
\end{aligned}
$$

Proof. The fact that $y(s)$ satisfies the differential inequality (2.12) follows immediately by the inequality (2.7) of lemma 2.5, by our choice of $p(s)$ and $q(s)$. Therefore $y(s) \leq y_{L}(s)$ for any $s \geq 0$ provided $y(0) \leq y_{L}(0)$ where $y_{L}(s)$ is a solution to:

$$
\frac{\mathrm{d}}{\mathrm{d} s} y_{L}(s)+p(s) y_{L}(s)+q(s)=0
$$

i.e.

$$
y_{L}(s)=\mathrm{e}^{-P(s)}\left[y_{L}(0)-\int_{0}^{s} q(\lambda) \mathrm{e}^{P(\lambda)} \mathrm{d} \lambda\right]=\mathrm{e}^{-P(s)}\left[y_{L}(0)-Q(s)\right]
$$

where

$$
P(s)=\int_{0}^{s} p(\lambda) \mathrm{d} \lambda, \quad Q(s)=\int_{0}^{s} q(\lambda) \mathrm{e}^{P(\lambda)} \mathrm{d} \lambda .
$$


Choosing $r(s)$ as in the statement one gets, after straightforward calculations,

$$
\mathrm{e}^{-P(t)}=\lim _{s \rightarrow t^{-}} \mathrm{e}^{-P(s)}=\frac{p r(0)+d(m(p-1)-1)}{r(0)} \frac{\varrho}{p \varrho+d(m(p-1)-1)}
$$

and

$$
\begin{aligned}
Q(t) & =\lim _{s \rightarrow t^{-}} \mathrm{Q}(\mathrm{s})=\frac{d}{p} \frac{\operatorname{pr}(0)+d(m(p-1)-1)}{r(0)}\left[\frac{-1}{p \varrho+d(m(p-1)-1)}\right. \\
& \left.+\frac{1}{p r(0)+d(m(p-1)-1)}\right] \log \left(\frac{m^{p-1} p^{p}}{d \mathcal{L}_{p}(\varrho-r(0))} t\right)+c_{1}(p, m, d, r(0), \varrho)
\end{aligned}
$$

for a suitable numerical constant $c_{1}(p, m, d, r(0), \varrho)$.

End of proof of Theorem 1.1. First we prove the claim for a $C_{c}^{\infty}(M)$ initial datum. Then the stated supercontractive bound (1.3) and ultracontractive bound (1.5) follows setting $r(0)=q$, noticing that $r(t)=\varrho$ and using the fact that each $\mathrm{L}^{q}$ norm of the solution is decreasing in time. Using the fact that the solution at all times belongs to $\mathrm{L}^{\infty}$, one has indeed

$$
\begin{aligned}
F^{\prime}(\tau) & =\frac{\mathrm{d}}{\mathrm{d} \tau}\|u(\tau)\|_{r}^{r} \\
& =-\frac{r(r-1) m^{p-1} p^{p}}{(r+m(p-1)-1)^{p}} \int_{M}\left|\nabla\left(|u(\tau, x)|^{\frac{r+m(p-1)-1}{p}}\right)\right|^{p} \mathrm{~d} x \leq 0
\end{aligned}
$$

and therefore

$$
\log \|u(t)\|_{\varrho}=\lim _{s \rightarrow t^{-}} \log \|u(t)\|_{r(s)} \leq \lim _{s \rightarrow t^{-}} \log \|u(s)\|_{r(s)}=\lim _{s \rightarrow t^{-}} y(s)
$$

So finally one obtains:

$$
\log \|u(t)\|_{\varrho} \leq \lim _{s \rightarrow t^{-}} y(s) \leq \lim _{s \rightarrow t^{-}} y_{L}(s)=y_{L}(t)
$$

that becomes:

$$
\begin{aligned}
\log \|u(t)\|_{\varrho} \leq & \frac{q}{\varrho} \frac{p \varrho+d(m(p-1)-1)}{p q+d(m(p-1)-1)}\|u(0)\|_{q} \\
& -\frac{d}{\varrho} \frac{(\varrho-q)}{p q+d(m(p-1)-1)} \log (t)+E\left(p, m, d, \mathcal{L}_{p}, q, \varrho\right)
\end{aligned}
$$

Taking now the exponentials of both members gives the supercontractivity inequality (1.3), valid for all $\varrho \geq 1$ and for all smooth, compactly supported data. The quantity $E$ above can be calculated explicitly by tedious but completely straightforward computations, and it is then easy to show that it admits a finite limit as $\varrho \rightarrow+\infty$. 
Letting $\varrho \rightarrow+\infty$ in the above inequality (2.16) then gives us:

$$
\begin{aligned}
\log \|u(t)\|_{\infty} \leq & \frac{p q}{p q+d(m(p-1)-1)} \log \|u(0)\|_{q} \\
& -\frac{d}{p q+d(m(p-1)-1} \log (t)+E\left(p, m, d, \mathcal{L}_{p}, q\right) .
\end{aligned}
$$

Taking the exponential of both members gives the ultracontractivity inequality (1.5) for all data $u_{0} \in C_{c}^{\infty}(M)$. Therefore for the data at hand the contractivity property:

$$
\|u(t)\|_{\infty} \leq\left\|u_{0}\right\|_{\infty}
$$

can then be obtained letting $q \rightarrow+\infty$.

Now we remove the assumption of boundedness of the datum $u_{0} \in \mathrm{L}^{q}$. To this end consider a sequence of functions $u_{k} \in C_{c}^{\infty}(M)$, converging to $u_{0}$ as $k \rightarrow+\infty$ in $\mathrm{L}^{q}$. Let $u_{k}(t)$ be the weak solution corresponding to the datum $u_{k}$. Then our bounds show that

$$
\left\|u_{k}(t)\right\|_{\varrho} \leq \frac{C\left\|u_{k}\right\|_{q}^{\gamma}}{t^{\alpha}} \leq C
$$

This implies that the sequence $u_{k}(t)$ is bounded in $\mathrm{L}^{\varrho}$ (for all $\varrho \in(q,+\infty]$ ) and hence weakly convergent (or weakly* convergent, if $\varrho=+\infty$ ) to a limit, say $v(t)$. We claim that $v(t)=u(t)$ for any $t>0$, where $u(t)$ is the weak solution to our problem corresponding to $u_{0}$. This follows from the continuous dependence on the data in the appropriate topologies proved in [5]). That $u(t)$ satisfies the stated bound is then a consequence of the lower semicontinuity, in the appropriate topology, of the norms involved. The other (simpler) statements follow similarly for general data as well.

To prove the fact that the exponents are the only possible for a bound of such a type we use a scaling argument. In fact, if $u(x, t)$ is a solution of the equation at hand and $c>0$ is given, then

$$
v_{1}(x, t)=c u\left(c^{m(p-1)-1} t, x\right)
$$

is a solution as well, corresponding to the initial datum $c u(0, x)$. Then the bound (1.3) applied to $v_{1}$ can be written as

$$
\left\|u\left(c^{m(p-1)-1} t\right)\right\|_{\varrho} \leq \frac{C}{t^{\alpha}} c^{\gamma-1}\|u(0)\|_{q}^{\gamma}
$$

so that, setting $c^{m(p-1)-1} t=s$ one must have

$$
\|u(s)\|_{\varrho} \leq \frac{C}{s^{\alpha}} c^{\gamma-1+\alpha[m(p-1)-1]}\|u(0)\|_{q}^{\gamma} \quad \forall c>0
$$


and hence the condition

$$
\gamma-1+\alpha[m(p-1)-1]=0
$$

must hold. Similarly, the function $v_{2}(x, t)=u\left(c^{p} t, c x\right)$ is a solution of the equation considered, in the spatial domain $c^{-1} M$. The corresponding initial datum is $u(0, c x)$. The bound (1.3) applied to $v_{2}$ then reads, after a change of spatial variables,

$$
\frac{1}{c^{d / \varrho}}\left\|u\left(c^{p} t\right)\right\|_{L^{\varrho}(D)} \leq \frac{C}{t^{\alpha}} c^{-d \gamma / q}\|u(0)\|_{L^{\varrho}(D)}^{\gamma}
$$

so that, setting $s=c^{p} t$, one must have

$$
\|u(s)\|_{\varrho} \leq \frac{C}{s^{\alpha}} c^{\frac{d}{\varrho}-\frac{d \gamma}{q}+p \alpha}\|u(0)\|_{q}^{\gamma} \forall c>0 .
$$

The condition

$$
\frac{d}{\varrho}-\frac{d \gamma}{q}+p \alpha=0
$$

must then hold as well. It is immediate to check that the only solutions to the couple of equalities just proved are the constants $\alpha, \gamma$ given in the statement. The proof is similar when the ultracontractive bound (1.5) is involved.

Proof of Theorem 1.2. We split the proof in the two stated cases, namely $m(p-1)>1$ and $m(p-1)=1$. In both cases we will use the Poincarè inequality:

$$
\|f\|_{p}^{p} \leq \lambda_{p}^{-1}\|\nabla f\|_{p}^{p}
$$

In both cases it suffices to prove the bounds for $C_{c}^{\infty}$ data, as discussed at the end of the proof of the first Theorem.

The case $\boldsymbol{m}(\boldsymbol{p}-1)>1$ : Hölder and Poincarè inequalities can be used to obtain a closed differential inequality which reads:

$$
\begin{aligned}
\frac{\mathrm{d}}{\mathrm{d} t}\|u(t)\|_{r}^{r} & =-\frac{r(r-1) m^{p-1} p^{p}}{(r+m(p-1)-1)^{p}}\left\|\nabla\left(|u(t)|^{\frac{r+m(p-1)-1}{p}}\right)\right\|_{p}^{p} \\
& \leq-\frac{r(r-1) m^{p-1} p^{p}}{(r+m(p-1)-1)^{p}} \lambda_{p}\left\||u(t)|^{\frac{r+m(p-1)-1}{p}}\right\|_{p}^{p} \\
& =-\frac{r(r-1) m^{p-1} p^{p}}{(r+m(p-1)-1)^{p}} \lambda_{p}\|u(t)\|_{r+m(p-1)-1}^{r+m(p-1)-1} \\
& =-\frac{r(r-1) m^{p-1} p^{p}}{(r+m(p-1)-1)^{p}} \lambda_{p} \operatorname{Vol}(M)^{-(m(p-1)-1) / r}\|u(t)\|_{r}^{r \frac{r+m(p-1)-1}{r}}
\end{aligned}
$$


so the function $\phi(t)=\|u(t)\|_{r}^{r}$, satisfies the differential inequality:

$$
\frac{\mathrm{d}}{\mathrm{d} t} \phi(t) \leq-C^{\prime} \phi(t)^{\frac{r+m(p-1)-1}{r}}
$$

that leads to:

$$
\|u(t)\|_{r} \leq \frac{1}{\left(C^{\prime} t+\left\|u_{0}\right\|_{r}^{-(m(p-1)-1)}\right)^{1 /(m(p-1)-1)}}
$$

with

$$
\begin{aligned}
C^{\prime} & =\frac{m(p-1)-1}{r} C \\
& =\frac{(m(p-1)-1)}{r} \frac{r(r-1) m^{p-1} p^{p}}{(r+m(p-1)-1)^{p}} \lambda_{p} \operatorname{Vol}(M)^{-(m(p-1)-1) / r}
\end{aligned}
$$

Now we use the ultracontractive inequality (1.5) and the above inequality, together with the semigroup property:

$$
u(t)=T_{t} u_{0}=T_{t / 2} u(t / 2)
$$

and we obtain:

$$
\begin{aligned}
\|u(t)\|_{\infty} & \leq K_{0} \frac{\|u(t / 2)\|_{r}^{\frac{p r}{p r+d(m(p-1)-1)}}}{t^{\frac{d}{p r+d(m(p-1)-1)}}} \\
& \leq \frac{K_{1}}{t^{\frac{d}{p r+d(m(p-1)-1)}}} \frac{1}{\left(C^{\prime} t+\left\|u_{0}\right\|_{r}^{-(m(p-1)-1)}\right)^{\frac{1}{(m(p-1)-1)} \frac{p r}{p r+m(p-1)-1}}}
\end{aligned}
$$

A simple numerical inequality will give us the absolute bound (1.8):

$$
\|u(t)\|_{\infty} \leq \frac{K_{3}}{t^{1 /(m(p-1)-1)}}
$$

The case $\boldsymbol{m}(\boldsymbol{p}-1)=1$ : we proceed as in the previous case (but we do not need Hölder inequality) to get a closed differential inequality that reads:

$$
\begin{aligned}
\frac{\mathrm{d}}{\mathrm{d} t}\|u(t)\|_{r}^{r} & =-\frac{r(r-1) m^{p-1} p^{p}}{r^{p}}\left\|\nabla\left(|u(t)|^{\frac{r}{p}}\right)\right\|_{p}^{p} \\
& \leq-\frac{(r-1) m^{p-1} p^{p}}{r^{p-1}} \lambda_{p}\|u(t)\|_{r}^{r}
\end{aligned}
$$

in this case the function $\phi(t)=\|u(t)\|_{r}^{r}$, satisfies the differential inequality:

$$
\frac{\mathrm{d}}{\mathrm{d} t} \phi(t) \leq-C \phi(t)
$$


that leads to:

$$
\|u(t)\|_{r} \leq \mathrm{e}^{-\frac{C}{r} t}\|u(0)\|_{r}=\mathrm{e}^{-\sigma t}\|u(0)\|_{r}
$$

with

$$
C=\frac{(r-1) m^{p-1} p^{p}}{r^{p-1}} \lambda_{p}
$$

Again by using the ultracontractivity inequality (1.5) together with the semigroup property, one gets:

$$
\|u(t)\|_{\infty} \leq K_{0} \frac{\|u(t / 2)\|_{r}}{t^{d / p r}} \leq K_{1} \frac{\mathrm{e}^{-\sigma t}}{t^{d / p r}}\|u(0)\|_{r}
$$

This concludes the proof.

\section{References}

[1] Bakry, D., Coulhon, T., Ledoux, M. and Saloff-Coste, L.: Sobolev inequalities in disguise. Indiana Univ. Math. J. 44 (1995), no. 4, 1033-1074.

[2] Bamberger, A.: Étude d'une équation doublement non linéaire. J. Functional Analysis 24 (1977), 148-155

[3] Benilan, P. and Crandall, M.G.: Completely Accretive Operators. In Semigroup Theory and Evolution Equations (Delft, 1989), 41-75. Lecture Notes in Pure and Applied Mathematics 135. M. Dekker, New York, 1991.

[4] Beckner, W.: Geometric asymptotics and the logaritmic Sobolev inequality. Forum Math. 11 (1999), 105-137

[5] Bernis, F.: Existence results for doubly nonlinear higher order parabolic equations on unbounded domains. Math. Ann. 279 (1988), 373-394

[6] Blanchard, D. and Francfort, G.A.: Study of a doubly nonlinear heat equation with no growth assumptions on the parabolic term. SIAM J. Math. Anal. 19 (1988), 1032-1056.

[7] Bonforte, M., Cipriani, F. and Grillo, G.: Ultracontractivity and convergence to equilibrium for supercritical quasilinear parabolic equations on Riemannian manifolds. Adv. Differential Equations 8 (2003), 843-872.

[8] Bonforte, M. and Grillo, G.: Asymptotics of the porous media equation via Sobolev inequalities. J. Funct. Anal. 225 (2005), 33-62.

[9] BÉnilan, P. and Picard, C.: Quelques aspects non linéaires du principe du maximum. In Séminaire de Théorie du Potentiel 4, 1-37. Lecture Notes in Math. 713, Springer, Berlin, 1979.

[10] Caisheng, C.: Global existence and $\mathrm{L}^{\infty}$ estimates of solution for doubly nonlinear parabolic equation. J. Math. Anal. Appl. 244 (2000), 133-146. 
[11] Cipriani, F. and Grillo, G.: Uniform bounds for solutions to quasilinear parabolic equations. J. Differential Equations 177 (2001), 209-234.

[12] Cipriani, F. and Grillo, G.: Nonlinear Markov semigroups, nonlinear Dirichlet forms and applications to minimal surfaces. J. Reine Angew. Math. 562 (2003), 201-235.

[13] Cipriani, F. and Grillo, G.: $\mathrm{L}^{p}-\mathrm{L}^{\infty}$ Hölder continuity for quasilinear parabolic equations associated to Sobolev derivations. J. Math. Anal. Appl. 270 (2002), 267-290.

[14] Davies, E. B.: Heat Kernels and Spectral Theory. Cambridge Tracts in Mathematics 92. Cambridge University Press, Cambridge, 1990.

[15] Del Pino, M. And Dolbeault, J.: The optimal euclidean L $^{p}$-Sobolev logarithmic inequality. J. Funct. Anal. 197 (2003), 151-161.

[16] Del Pino, M. And Dolbeault, J.: Best constants for GagliardoNirenberg inequalities and applications to nonlinear diffusions. J. Math. Pures Appl. (9) 81 (2002), 847-875.

[17] Del Pino, M., Dolbeault, J. And Gentil, I.: Nonlinear diffusions, hypercontractivity and the optimal $\mathrm{L}^{p}$-Euclidean logarithmic Sobolev inequality. J. Math. Anal. Appl. 293 (2004), 375-388.

[18] Di Benedetto, E.: Continuity of weak solutions to a general porous medium equation. Indiana Univ. Math. J. 32 (1983), 83-118

[19] Ebmeyer, C. and Urbano, J.M.: Regularity in Sobolev spaces for doubly nonlinear parabolic equations. J. Differential Equations 187 (2003), 375-390.

[20] Filo, J.: Local existence and $\mathrm{L}^{\infty}$-estimate of weak solutions to a nonlinear degenerate parabolic equation with nonlinear boundary data. Panamer. Math. J. 4 (1994), 1-31

[21] Gentil, I.: The general optimal L ${ }^{p}$-Euclidean logarithmic Sobolev inequality by Hamilton-Jacobi equations. J. Funct. Anal. 202 (2003), 591-599.

[22] Gross, L.: Logarithmic Sobolev inequalities. Amer. J. Math. 97 (1975), 1061-1083.

[23] Ishige, K.: On the existence of solutions of the Cauchy problem for a doubly nonlinear parabolic equation. SIAM J. Math. Anal. 27 (1996), $1235-1260$.

[24] Lindqvist, P.: On nonlinear Rayleigh quotients. Potential Anal. 2 (1993), 199-218.

[25] Manfredi, J. J. And Vespri, V.: Large time behavior of solutions to a class of doubly nonlinear parabolic equations. Electron. J. Differential Equations 1994 (1994), approx. 17 pp. (electronic only).

[26] Porzio, M. M. And Vespri, V.: Hölder estimates for local solutions of some doubly nonlinear degenerate parabolic equations. J. Differential Equations 103 (1993), 146-178. 
[27] SAÁ, J.: Large time behaviour of the doubly nonlinear porous medium equation. J. Math. Anal. Appl. 155 (1991), 345-363.

[28] Tsutsumi, M.: On solutions of some doubly nonlinear degenerate parabolic equations with absorption. J. Math. Anal. Appl. 132 (1988), $187-212$.

Recibido: 25 de marzo de 2003

Matteo Bonforte Dipartimento di Matematica

Politecnico di Torino corso Duca degli Abruzzi 24

10129 Torino, Italy bonforte@calvino.polito.it

Gabriele Grillo

Dipartimento di Matematica

Politecnico di Torino corso Duca degli Abruzzi 24 10129 Torino, Italy gabriele.grillo@polito.it 